\title{
Real-time thermal field theory analyses of 2D Gross-Neveu model*
}

\author{
ZHOU Bangrong \\ Department of Physics, Graduate School at Beijing \\ University of Science and Technology of China, Academia Sinica, Beijing 100039, China \\ and \\ CCAST (World Laboratory), Beijing 100080, China
}

\begin{abstract}
Discrete symmetry breaking and possible restoration at finite temperature $T$ are analysed in 2D Gross-Neveu model by the real-time thermal field theory in the fermion bubble approximation. The dynamical fermion mass $m$ is proven to be scaleindependent and this fact indicates the equivalence between the fermion bubble diagram approximation and the mean field approximation used in the auxialiary scalar field approach. Reproducing of the non-zero critical temperature $T_{c}=0.567 \mathrm{~m}(0)$, $(m(0)$ is the dynamical fermion mass at $T=0)$, shows the equivalence between the real-time and the imaginary-time thermal field theory in this problem. However, in the real-time formalism, more results including absence of scalar bound state, the equation of criticality curve of chemical potential-temperature and the $\ln \left(T_{c} / T\right)$ behavior of $m^{2}$ at $T \lesssim T_{c}$ can be easily obtained. The last one indicates the second-order phase transition feature of the symmetry restoration.
\end{abstract}

PACS numbers: 11.10.Kk, 11.10.Wx, 11.30.Er, 11.30.Qc

Key words: 2D Gross-Neveu model, spontaneous discrete symmetry breaking, real-time thermal field theory, fermion bubble diagram approximation

\section{Introduction}

In 4D model of NJL-form [1] and 3D Gross-Neveu (GN) model [2], by means of analyses of dynamical fermion mass in the real-time thermal field theory in the fermion bubble approximation, we have acquired a quite clear understanding of restorations of the spontaneously broken symmetries at high temperature and their second-order phase transition feature [3,4]. A natural idea is to extend the same analysis to 2D GN model. However, once entering one-space dimension, one will find some special features. By the MerminWagner-Coleman Theorem [5], a continuous symmetry can never be spontaneously broken in 2 dimensions. Consequently we will have to confine ourselves to possible spontaneous breaking of only some discrete symmetries. The 2D GN model is perturbatively renormalizable and asymptotically free and this is apparently different from the perturbatively non-renormalizable 4D NJL model and 3D GN model. Based on the auxialiary scalar field

*This work was partially supported by National Natural Science Foundation of China and by Grant No. LWTZ-1298 of Chinese Academy of Sciences. 
approach, many researches on the behavior of 2D GN model at finite temperature have been made $[6,7,8]$, though all of them in the imaginary-time formalism of thermal field theory. Considering the above facts, in this paper, we will analysed 2D GN model in the real-time thermal field theory in the fermion bubble approximation. Through comparison between the results obtained by our approach and the known ones, we will be able to check the equivalence of the real-time and the imaginary-time formalism of thermal field theory in this problem, find the advantages of the real-time formalism and the limitations of the fermion bubble approximation and, in the meantime, get deeper insight into the 2D GN model itself as well.

The paper is arranged as follows. In Sect.2 we will analyse the symmetries of 2D GN model, mainly its discrete symmetries and their possible spontaneous breaking induced by dynamical fermion mass. In Sect. 3 the behavior of the model at temperature $T=0$ will be outlined. This includes derivation of the renormalized gap equation and the running coupling constant in dimension regularization scheme and the proof of that scalar bound state could not exist in this model. In Sect. 4 we extend the gap equation to the case of $T \neq 0$, determine the critical temperature of symmetry restoration and derive the critical behavior of the dynamical fermion mass. Finally in Sect. 5 we indicate the advantages and the limitations of our approach through the comparison with the known results and reach corresponding conclusions.

\section{Symmetry analysis of the model}

The Lagrangian of 2D GN model is expressed by

$$
\mathcal{L}=\sum_{k=1}^{N} \bar{\psi}^{k} i \gamma^{\mu} \partial_{\mu} \psi_{k}+\frac{g}{2} \sum_{k=1}^{N}\left[\bar{\psi}^{k} \psi_{k}\right]^{2} .
$$

where $\psi \equiv \psi_{k}(t, x)$ are the spinor fields with $N$ "color" components. In 2 dimesions, the four-fermion coupling constant $g$ is dimensionless thus the theory is perturbatively renormalizable. The $\gamma^{\mu}(\mu=0,1)$ and $\gamma_{5}=\gamma^{0} \gamma^{1}$ are $2 \times 2$ matrices which are related to the Pauli matrices $\sigma^{i}(i=1,2,3)$ by

$$
\gamma^{0}=\sigma^{1}=\left(\begin{array}{ll}
0 & 1 \\
1 & 0
\end{array}\right), \gamma^{1}=i \sigma^{2}=\left(\begin{array}{cc}
0 & 1 \\
-1 & 0
\end{array}\right) \quad \text { and } \gamma_{5}=-\sigma^{3}=\left(\begin{array}{cc}
-1 & 0 \\
0 & 1
\end{array}\right)
$$

i.e. we take the "Weyl" representation. In this representation, $\psi_{k}$ will be two-component complex spinors. We will concern only the discrete symmetries of the model, since continuous symmetries can not be spontaneously broken in 2 dimensions. It is easy to see that the Lagrangian (2.1) is invariant under the discrete transformation $R$,

$$
\psi(t, x) \stackrel{R}{\longrightarrow}-\psi(t, x)
$$

and the ordinary parity $\mathcal{P}$ and the time reversal $\mathcal{T}$,

$$
\begin{aligned}
& \psi(t, x) \stackrel{\mathcal{P}}{\longrightarrow} \gamma^{0} \psi(t,-x), \\
& \psi(t, x) \stackrel{\mathcal{T}}{\longrightarrow} \gamma^{0} \psi(-t, x) .
\end{aligned}
$$

In addition, it is also invariant under the discrete chiral transformation $\chi_{D}$,

$$
\psi(t, x) \stackrel{\chi_{D}}{\longrightarrow} \gamma_{5} \psi(t, x)
$$

and the special parity $\mathcal{P}_{1}$

$$
\psi(t, x) \stackrel{\mathcal{P}_{1}}{\longrightarrow} \gamma^{1} \psi(t,-x)
$$


We indicate that the fermion mass term $-m \bar{\psi} \psi$ will keep $R, \mathcal{P}$ and $\mathcal{T}$ invariant but not $\chi_{D}$ and $\mathcal{P}_{1}$ invariant. Therefore, the dynamical generation of the fermion mass will imply spontaneous breaking of the discrete chiral symmetry $\chi_{D}$ and the special parity $\mathcal{P}_{1}$. The spontaneous breaking of $\chi_{D}$ and $\mathcal{P}_{1}$ and their possible restoration at finite temperature $T$ is just the problem we will discussed.

\section{The behavior of the model at $T=0$}

The renormalization operation will be conducted in the dimension regularization approach [9]. For this purpose, we change the time-space dimension from 2 into

$$
D=2-2 \varepsilon
$$

and the Lagrangian $\mathcal{L}$ in Eq.(2.1) into

$$
\mathcal{L}_{D}=\sum_{k=1}^{N} \bar{\psi}^{k} i \gamma^{\mu} \partial_{\mu} \psi_{k}+\frac{g}{2} M^{2-D} \sum_{k=1}^{N}\left[\bar{\psi}^{k} \psi_{k}\right]^{2}
$$

where $M$ is a scale parameter with mass dimension, the mass dimensions $[g]=0$, $\left[\psi_{k}\right]=\left[\bar{\psi}^{k}\right]=(D-1) / 2$ and the dimension of the representation of the matrices $\gamma^{\mu}$ becomes $2^{D / 2}$. The Feynman rule of the four-fermion vertex will be $i g M^{2-D}$.

Assuming that the four-fermion interactions will lead to the condensates $\sum_{k=1}^{N}\left\langle\bar{\psi}^{k} \psi_{k}\right\rangle \neq$ 0 , then the non-zero dynamical mass

$$
m(0)=-g M^{2-D} \sum_{k=1}^{N}\left\langle\bar{\psi}^{k} \psi_{k}\right\rangle
$$

will be generated and the discrete symmetries $\chi_{D}$ and $\mathcal{P}_{1}$ will be spontaneously broken. After the Wick rotation and introduction of the denotations $\bar{l}^{0}=i l^{0}, \bar{l}^{i}=l^{i}$, we obtain from Eq.(3.3) the gap equation

$$
\begin{aligned}
1 & =g N 2^{D / 2} M^{2-D} \int_{-\infty}^{\infty} \frac{d^{D} \bar{l}}{(2 \pi)^{D}} \frac{1}{\bar{l}^{2}+m^{2}(0)} \\
& =g N 2^{D / 2} M^{2-D} \frac{\Gamma(1-D / 2)}{(4 \pi)^{D / 2}\left[m^{2}(0)\right]^{1-D / 2}}
\end{aligned}
$$

where we have used the integral formula [10]

$$
\int_{-\infty}^{\infty} \frac{d^{D} \bar{l}}{(2 \pi)^{D}} \frac{1}{\left[\bar{l}^{2}+m^{2}(0)\right]^{A}}=\frac{\Gamma(A-D / 2)}{(4 \pi)^{D / 2} \Gamma(A)\left[m^{2}(0)\right]^{A-D / 2}} .
$$

Considering Eq. (3.1), the gap equation can be reduced to

$$
1=\frac{g N}{2 \pi}\left[\frac{1}{\varepsilon}-\gamma+\ln \frac{2 \pi M^{2}}{m^{2}(0)}+\mathcal{O}(\varepsilon)\right]
$$

where $\gamma=0.5772$ is the Euler constant. The divergent $1 / \varepsilon$ term can be removed by introduction of the counter-term of the four-fermion interactions

$$
\mathcal{L}_{\text {c.t. }}=\frac{g}{2} M^{2-D}\left(Z_{4}-1\right) \sum_{k=1}^{N}\left(\bar{\psi}^{k} \psi_{k}\right)^{2} .
$$


The renormalization constant $Z_{4}$ is defined by the minimal subtraction scheme [9], i.e., to one-loop order,

$$
Z_{4}=1-\frac{g N}{2 \pi} \frac{1}{\varepsilon}
$$

then the renormalized gap equation will take the form

$$
1=\frac{g N}{2 \pi}\left[\ln \frac{2 \pi M^{2}}{m^{2}(0)}-\gamma\right]
$$

Eq. (3.9) gives that

$$
m^{2}(0)=2 \pi M^{2} \exp \left[-\frac{2 \pi}{g N}-\gamma\right]
$$

Since 2D GN model is an asymptotically free theory, as will be argued immediately, the $g$ in Eq. (3.10) should be replaced by corresponding running coupling constant. The running coupling can be derived by the general renormalization group method. In fact, after introducing the counter-term $\mathcal{L}_{\text {c.t. }}$, we can define the bare four-fermion coupling constant

$$
g_{0}=g M^{2-D} Z_{4}
$$

which should be independent of the renormalization scale parameter M, i.e.

$$
\frac{d g_{0}}{d M}=0
$$

Considering Eq. (3.8), Eq. (3.12) will lead to

$$
M \frac{\partial g}{\partial M}=-\frac{N}{\pi} g^{2}
$$

and furthermore,

$$
\frac{1}{g(M)}=\frac{N}{\pi} \ln \frac{M}{M_{0}}+\frac{1}{g\left(M_{0}\right)}
$$

Eq. (3.14) indicates that the running coupling $g(M) \rightarrow 0$ when $M \rightarrow \infty$, i.e. the theory is ultraviolet stable and asymptotically free. Now we replace the coupling constant $g$ in Eq. (3.10) by the running coupling $g(M)$, then by means of Eq. (3.14), can prove that

$$
m^{2}(0)=2 \pi M^{2} \exp \left[-\frac{2 \pi}{g(M) N}-\gamma\right]=2 \pi M_{0}^{2} \exp \left[-\frac{2 \pi}{g\left(M_{0}\right) N}-\gamma\right]
$$

i.e. the dynamical fermion mass $m(0)$ is scale-indepedent, we will obtain the same $m(0)$ in the scales $M$ and $M_{0}$. On the other words, the order parameter $m(0)$ of the discrete symmetry breaking will be independent of the size of the system.

We can also calculate the renormalized fermionic four-point function $\Gamma_{S}^{R}(p)$ in scalar channel by the methods used in Ref. [11]. The result is

$$
\begin{gathered}
\Gamma_{S}^{R}(p)=-i /\left[p^{2}-4 m^{2}(0)+i \varepsilon\right] K_{0}(p), \\
K_{0}(p)=\frac{N}{4 \pi} \int_{0}^{1} \frac{d x}{m^{2}(0)-p^{2} x(1-x)}=\frac{N}{\pi p^{2} \sqrt{4 m^{2}(0) / p^{2}-1}} \arctan \frac{1}{\sqrt{4 m^{2}(0) / p^{2}-1}},
\end{gathered}
$$

where we have made the assumption that $0<p^{2}<4 m^{2}(0)$. To examine if $p^{2}=4 m^{2}(0)$ could be a simple pole of $\Gamma_{S}^{R}(p)$, we may calculate the residual of $\Gamma_{S}^{R}(p)$ at $p^{2}=4 m^{2}(0)$ and obtain

$$
\lim _{p^{2} \rightarrow 4 m^{2}(0)}\left[\frac{4 m^{2}(0)}{p^{2}}-1\right] \Gamma_{S}^{R}(p)=0
$$


which implies that $\Gamma_{S}^{R}(p)$ does not represent a propogator for any scalar bound state with the mass $2 m(0)$. Hence, the scalar bound state does not exist at $T=0$ in this model. In fact, this conclusion can be generalized to the case of $T \neq 0$, as will be showed in the next Section.

\section{Symmetry restoration and phase transition at fi- nite temperature}

We will take the real-time formalism of thermal field theory [12] to research the temperature behavior of the dynamical fermion mass. This means that, corresponding to the vacuum expectation value $\sum_{k}\left\langle\bar{\psi}^{k} \psi_{k}\right\rangle$ replaced by the thermal expectation value $\sum_{k}\left\langle\bar{\psi}^{k} \psi_{k}\right\rangle_{T}$, we should make the substitution of the fermion propagator

$$
\begin{gathered}
\frac{i}{V-m+i \varepsilon} \longrightarrow \frac{i}{V-m+i \varepsilon}-2 \pi \delta\left(l^{2}-m^{2}\right)(y+m) \sin ^{2} \theta\left(l^{0}, \mu\right), \\
\sin ^{2} \theta\left(l^{0}, \mu\right)=\frac{\theta\left(l^{0}\right)}{\exp \left[\beta\left(l^{0}-\mu\right)\right]+1}+\frac{\theta\left(-l^{0}\right)}{\exp \left[\beta\left(-l^{0}+\mu\right)\right]+1},
\end{gathered}
$$

where $\mu$ is chemical potential and $\beta=1 / T$. Let $m \equiv m(T, \mu)$ is the dynamical fermion mass at finite temperature $T$ and finite chemical potential $\mu$, then similar to the derivation at $T=0$, we may obtain the gap equation at $T \neq 0$

$$
1=Z_{4} \frac{g N}{2 \pi}\left[\frac{1}{\varepsilon}-\gamma+\ln \frac{2 \pi M^{2}}{m^{2}}-2 \int d^{2} l \delta\left(l^{2}-m^{2}\right) \sin ^{2} \theta\left(l^{0}, \mu\right)\right]
$$

Notice that the integral related to $T$ is convergent and the UV divergence appears only in the zero temperature sector. Therefore, by the definition (3.8) of $Z_{4}$ at $T=0$, we can remove the $1 / \varepsilon$ divergence to the one-loop order and obtain the renormalized gap equation at $T \neq 0$

$$
1=\frac{g N}{2 \pi}\left\{\ln \frac{2 \pi M^{2}}{m^{2}}-\gamma-2\left[I_{1}(y,-r)+I_{1}(y, r)\right]\right\}
$$

where

$$
I_{1}(y, \mp r)=\int_{0}^{\infty} \frac{d x}{\sqrt{x^{2}+y^{2}}} \frac{1}{\exp \left(\sqrt{x^{2}+y^{2}} \mp r\right)+1}
$$

with the denotations $y=m / T$ and $r=\mu / T$. By the same argument as the one leading to Eq. (3.15), it can be proven that the dynamical fermion mass $m$ at finite temperature and chemical potential is also scale-independent. This implies that in the bubble fermion diagram approximation, the order parameter is always size-independent.

Substitute the gap equation (3.9) at $T=0$ into Eq. (4.3), we will obtain

$$
\ln \frac{m(0)}{m}=I_{1}(y,-r)+I_{1}(y, r)
$$

We may assume that there is a critical temperature $T_{c} \neq 0$ so that when $T \rightarrow T_{c}, m \rightarrow 0$. It is easy to see that this assumption is consistent with Eq. (4.5). In fact, when $m \rightarrow 0$, in the left-handed side of Eq. (4.5) there will appear $\ln \infty$ divergence and in the righthanded side, the integral at $x \rightarrow 0$ will approach $\int_{0}^{\infty} \frac{d x}{x}$ and has the same $\ln \infty$ behavior. Thus the assumption $T_{c} \neq 0$ is plausible, at least in the bubble diagram approximation. 
Since when $T \lesssim T_{c} \neq 0, m \sim 0$, i.e. $y \ll 1$, we can use the high temperature expansion of $I_{1}(y, \mp r)[3]$ and obtain from Eq. (4.5)

$$
\begin{gathered}
\ln \frac{m(0)}{T \pi}+\gamma=\frac{7}{2} \zeta(3)\left(\frac{y}{2 \pi}\right)^{2}+\left[7 \zeta(3)-93 \zeta(5)\left(\frac{y}{2 \pi}\right)^{2}\right]\left(\frac{r}{2 \pi}\right)^{2} \\
+\left[-31 \zeta(5)+\frac{1905}{2} \zeta(7)\left(\frac{y}{2 \pi}\right)^{2}\right]\left(\frac{r}{2 \pi}\right)^{4}+127 \zeta(7)\left(\frac{r}{2 \pi}\right)^{6}+\mathcal{O}\left[\left(\frac{y}{2 \pi}\right)^{4}\right]
\end{gathered}
$$

where $\zeta(3)=1.202, \zeta(5)=1.037, \ldots$ and $r / 2 \pi<1$ is also assumed. We find that the $\ln m$ terms in both sides of Eq. (4.6) have cancelled each other. Taking $y=0$ i.e. $m=0$, we will obtain the equation of $T_{c}$ and the critical chemical potential $\mu_{c}$

$$
\ln \frac{m(0)}{T_{c} \pi}+\gamma=7 \zeta(3)\left(\frac{r_{c}}{2 \pi}\right)^{2}-31 \zeta(5)\left(\frac{r_{c}}{2 \pi}\right)^{4}+127 \zeta(7)\left(\frac{r_{c}}{2 \pi}\right)^{6}+\cdots
$$

with the denotation $r_{c}=\mu_{c} / T_{c}$. When chemical potential is zero, Eq. (4.7) gives

$$
T_{c}=\left(e^{\gamma} / \pi\right) m(0)=0.567 m(0)
$$

This result is identical to the one obtained in the mean field approximation of the auxialiary scalar field approach in the imaginary-time formalism of thermal field theory [6-8]. When the chemical potential is not equal to zero, Eq. (4.7) will be able to determine a $\mu_{c}-T_{c}$ criticality curve at $r_{c} / 2 \pi<1$. This shows an advantage of the real-time thermal field theory approach. Besides this, we may further give the critical behavior of the dynamical fermion mass $m$ i.e. the order parameter of symmetry breaking at $T \lesssim T_{c}$. Since $y \ll 1$ when $T \lesssim T_{c}$, it is reasonable to keep only the terms of the order $\mathcal{O}\left(y^{2}\right)$ in Eq. (4.6). Then, considering the equation (4.7) of $\mu_{c}-T_{c}$ curve (in which $\mu_{c}$ is redenoted by $\mu$ ), we may derive the expression for the squared dynamical fermion mass

$$
\begin{gathered}
m^{2}=4 \pi^{2} T^{2}\left[\ln \frac{T_{c}}{T}+7 \zeta(3)\left(\frac{\mu}{2 \pi}\right)^{2}\left(\frac{1}{T_{c}^{2}}-\frac{1}{T^{2}}\right)-31 \zeta(5)\left(\frac{\mu}{2 \pi}\right)^{4}\left(\frac{1}{T_{c}^{4}}-\frac{1}{T^{4}}\right)\right. \\
\left.+127 \zeta(7)\left(\frac{\mu}{2 \pi}\right)^{6}\left(\frac{1}{T_{c}^{6}}-\frac{1}{T^{6}}\right)\right] /\left[\frac{7}{2} \zeta(3)-93 \zeta(5)\left(\frac{\mu}{2 \pi}\right)^{2} \frac{1}{T^{2}}+\frac{1905}{2} \zeta(7)\left(\frac{\mu}{2 \pi}\right)^{4} \frac{1}{T^{4}}\right], \\
\text { when } T \lesssim T_{c} .
\end{gathered}
$$

If the chemical potential is zero, then Eq. (4.9) gives

$$
m^{2}=\frac{8 \pi^{2}}{7 \zeta(3)} T^{2} \ln \frac{T_{c}}{T}, \text { when } T \lesssim T_{c} .
$$

The logrithmic behavior of $m^{2}$ for $T_{c}$ can be compared with the temperature behaviors of $m^{2}$ at $T \lesssim T_{c}$ in $4 \mathrm{D}$ NJL model and 3D GN model. The latters are [3,4]

$$
\begin{gathered}
m^{2}=\frac{\pi^{2}}{3} \frac{T_{c}^{2}-T^{2}}{\ln (\Lambda / T \pi)+\gamma-1 / 2}, \text { for } 4 \mathrm{D} \text { NJL model } \\
m^{2}=8(\ln 2) T\left(T_{c}-T\right), \text { for 3D GN model }
\end{gathered}
$$

In both cases we have $m \sim\left(T_{c}-T\right)^{1 / 2}$ at $T \lesssim T_{c}$ and it means that the relevant symmetry restorations are the second-order phase transitions. In the case of $2 \mathrm{D}$ GN model, based on a similar argument to the one given in Ref. [3], we can reach the same conclusion, i.e. the discrete symmetry restoration at $T>T_{c}$ is also the second-order phase transition. 
Based the method used in Ref. [13], we can obtain the renormalized fermionic fourpoint function $\Gamma_{S T}^{R}(p)$ in scalar channel at finite temperature $T$ which is expressed by

$$
\Gamma_{S T}^{R}(p)=-i /\left(p^{2}-4 m^{2}+i \varepsilon\right)\left\{K(p)+H(p)-i S(p)-R^{2}(p) /[K(p)+H(p)+i S(p)]\right\},
$$

where $K(p)$ has the same form as $K_{0}(p)$ in Eq. (3.17), except that $m(0)$ must be replaced by $m$, and

$$
\begin{gathered}
H(p)=\frac{N}{2 \pi} \int d^{2} l\left[\frac{p^{2}-2 l \cdot p}{\left(p^{2}-2 l \cdot p\right)^{2}+\varepsilon^{2}}+\frac{p^{2}+2 l \cdot p}{\left(p^{2}+2 l \cdot p\right)^{2}+\varepsilon^{2}}\right] \delta\left(l^{2}-m^{2}\right) \sin ^{2} \theta\left(l^{0}, \mu\right), \\
S(p)=\frac{N}{2} \int d^{2} l \delta\left(l^{2}-m^{2}\right) \delta\left[(l+p)^{2}-m^{2}\right]\left[\sin ^{2} \theta\left(l^{0}, \mu\right) \cos ^{2} \theta\left(l^{0}+p^{0}, \mu\right)+\cos ^{2} \theta\left(l^{0}, \mu\right) \sin ^{2} \theta\left(l^{0}+p^{0}, \mu\right)\right] \\
R(p)=\frac{N}{4} \int d^{2} l \delta\left(l^{2}-m^{2}\right) \delta\left[(l+p)^{2}-m^{2}\right] \sin 2 \theta\left(l^{0}, \mu\right) \sin 2 \theta\left(l^{0}+p^{0}, \mu\right)
\end{gathered}
$$

The form (4.13) of $\Gamma_{S T}^{R}(p)$ ensures mutual cancellation of the pinch singularities contained in $S(p)$ and $R(p)$. It is easy to confirm that when $p^{2} \rightarrow m^{2}, H(p)$ is finite but $S(p)$ and $R(p)$ are sigular. The latter comes from the feature of the product of the two $\delta$-functions. Since $K(p)$ is divergent when $p^{2} \rightarrow m^{2}$, we will still have the residual of $\Gamma_{S T}^{R}(p)$ at $p^{2}=4 m^{2}$

$$
\lim _{p^{2} \rightarrow 4 m^{2}}\left(\frac{4 m^{2}}{p^{2}}-1\right) \Gamma_{S T}^{R}(p)=0
$$

Hence, the conclusion that there is no scalar bound state in the model will be maintained at finite temperature.

Our results about finite temperature behavior of the model are obtained by the realtime thermal field theory in the fermion bubble diagram approximation. It should be pointed that the fermion bubble diagram approximation has already been able to reflect physical essentiality in 4D and 3D case. However, it has great limitations in 2D case. As illustrated in Ref. [8], where the auxialiary scalar field approach was used, for an infinite one-space system and a finite $N$, in fact there is not symmetry restoration at $T \neq 0$ owing to the special kink effects when the minimal configuration $\sigma_{c}(x)$ of the effective thermodynamic potential $\Gamma\left(\sigma_{c}(x), \beta\right)$ could be a function of space. Only if $\sigma_{c}(x)=$ constant is assumed, i.e. in so called the mean field approximation, one could obtain symmetry restoration at $T \neq 0$ and this is just the result obtained in Refs. [6,7] and in this paper. It may be indicated that the scale-independece, or the size-independence of the order parameter $m(T, \mu)$ proved in this paper is just consistent with the assumption $\sigma_{c}(x)=$ constant in the mean field approximation. Hence we can say that the fermion bubble approximation is indeed the mean field approximation named in Ref. [8]. Since the mean field approximation is valid only if $N \rightarrow \infty$ or if $N$ is large and one consider only a finite segment of one-space dimension system, the results about finite temperature obtained in this paper are applicable only in the above conditions.

\section{Conclusions}

We have analysed spontaneous breaking and restoration of the discrete chiral symmetry $\chi_{D}$ and the special parity $\mathcal{P}_{1}$ induced by dynamical fermion mass in renormalizable and asymptotically free 2D Gross-Neveu model by means of the real-time thermal field theory in the fermion bubble diagram approximation. We have proven that the dynamical fermion mass, both $m(0)$ at zero-temperature and $m$ at finite temperature, are scale-independent, consistent with the fundamental assumption of the mean field approximation in the auxialiary scalar field approach. Hence, the fermion bubble diagram 
approximation is equivalent to the mean field approximation and must submit to the same applicable conditions. All the results, especially reproducing of the non-zero symmetry restoration temperature $T_{c}=0.567 \mathrm{~m}(0)$, show the equivalence between the real-time and the imaginay-time formalism of thermal field theory in this problem. However, in the real-time formalism, one can further prove that there is no scalar bound state in the model, easily obtain the equation of criticality curve of chemical potential and temperature and the $\ln \left(T_{c} / T\right)$ behavior of the dynamical squared fermion mass $m^{2}$ at $T \lesssim T_{c}$. The last one clearly indicates the second-order phase transition feature of the symmetry restorations.

\section{References}

[1] Y. Nambu and G. Jona-Lasinio, Phys. Rev. 122 (1961) 345; 124 (1961) 246.

[2] D. J. Gross and A. Neveu, Phys. Rev. D10 (1974) 3235.

[3] B. R. Zhou, Phys. Rev. D57 (1998) 3171; Commun. Theor. Phys. 32 (1999) 425.

[4] B. R. Zhou, Phys. Lett. B444 (1998) 455.

[5] N. D. Mermin and H. Wagner, Phys. Rev. Lett. 17 (1966) 1133; S. Coleman, Commun. Math. Phys. 31 (1973) 259.

[6] L. Jacobs, Phys. Rev. D10 (1974) 3956.

[7] B. J. Harrington and A. Yildiz, Phys. Rev. D11 (1975) 779.

[8] R. F. Dashen, S-K, Ma and R. Rajaraman, Phys. Rev. D11 (1975) 1499.

[9] D. J. Gross, Application of the renormalization group, in Methods in Field Theory, eds. R. Balian and J. Zinn-Justin, North-Holland (1976) p. 140.

[10] P. Ramond, Field Theory - a Modern Primer 2nd ed., Addison-Wesley Publishing Company, Inc. (1989).

[11] B. R. Zhou, Phys. Rev. D47 (1993) 5038.

[12] N. P. Landsman and van Ch. G Weert, Phys. Rep. 145 (1987) 141 and references therein.

[13] B. R. Zhou, Commun. Theor. Phys.,33 (2000) 113. 